\title{
A Study on Measuring Distribution of Temperature for Instrumented Taylor Impact Test
}

\author{
Chong $\mathrm{Gao}^{1}$, and Takeshi Iwamoto ${ }^{2 *}$ \\ ${ }^{1}$ Graduate School of Engineering, Hiroshima University, 1-4-1 Kagamiyama, Higashi-Hiroshima, \\ 739-8527 Japan \\ ${ }^{2}$ Academy of Science and Technology, Hiroshima University, 1-4-1 Kagamiyama, Higashi- \\ Hiroshima, Hiroshima, 739-8527 Japan
}

\begin{abstract}
It is a challenge to apply the infrared detector to a test at higher strain rate because lots of articles report that the detector has an extremelyhigh responsiveness. However, all the research works about the measurement of temperature is focused on $10^{3} \mathrm{~s}^{-1}$ of the strain rate achieved by the split Hopkinson pressure bar test. To evaluate the thermo-mechanical behaviour of materials in higher strain rate range, a method for measuring the temperature in Taylor impact test is required to establish. Additionally, a use of the fiber should be considered to protect the detector from any damages by the impact of the specimen and realize the measurement in the point-like area. In this study, an optically-measuring system of temperature with the infrared detector and PIR fiber is designed. Then, the designed system is introduced into the apparatus based on the instrumented Taylor impact test proposed recently. The temperature rise at a local point on the surface of pure aluminium is measured during the test. Then, a calculation for distribution of temperature from a heat conduction equation is proposed.
\end{abstract}

\section{Introduction}

In our previous study, an instrumented Taylor impact test is established [1] for measuring just a stress-strain curve at ultra-high strain rate over $10^{5} \mathrm{~s}^{-1}$. Focusing on the non-uniform deformation of the specimen, the test is instrumented by a high-speed camera [2] and Hopkinson pressure bar [3] to combine obtained distribution of axial strain and stress. Additionally, because the deformation rate is much higher than the release rate of internallydeveloped heat during high-speed deformation, the adiabatic process and a significant rise in temperature of specimen are achieved. Thus, when performing the Taylor impact test, not only the mechanical but also the thermal behaviour of the materials should be evaluated together because the thermal softening gets significant due to the temperature rise under such high-speed deformation.

In general, the thermocouple and infrared detector are widely used for evaluating the thermal behaviour of the materials. In previous, lots of challenges on measuring the

* Corresponding author: iwamoto@mec.hiroshima-u.ac.jp 
temperature rise in high strain rate range is done by using the extremely-thin thermocouple [4-6] and infrared detector [7]. Their high responsiveness is confirmed from the measurement result. Additionally, it can be predicted that the responsiveness of infrared detector is much higher than thermocouple because of its nature of light. So that, the infrared detector is applied to determine the Taylor-Quinney coefficient [8] of materials at high strain rate frequently [9-11]. From the viewpoint, it is possible to apply the infrared detector to measure the temperature rise of materials in a higher strain rate range because of its responsiveness.

However, all the past measurements of temperature rise are conducted at the strain rate around $10^{3} \mathrm{~s}^{-1}$ by carrying out split Hopkinson pressure bar (SHPB) test. Thus, measuring temperature at over $10^{5} \mathrm{~s}^{-1}$ of the strain rate need to be realized. When the temperature is measured during the Taylor impact test, a non-contact technique such as the infrared detector is primally introduced. Generally speaking, the infrared detector can capture the temperature in the spot-like small area with the same size of a detecting sensor on the surface of the specimen. A fiber should be introduced to prevent any damage of the infrared detector due to a shot specimen and realize the measurement in the point-like area smaller than the detection area of the device. Different from the SHPB test with uniform deformation, the temperature in only the area is insufficient to evaluate the thermal behaviour which should be focused on due to a nature of the non-uniform deformation in the specimen.

In this study, measuring distribution of temperature during Taylor impact test is established. At first, an optical system including the infrared detector and PIR fiber is designed to capture the temperature rise at one spot with diameter of fiber. After that, the designed optical system is introduced into the apparatus based on the instrumented Taylor impact test. A Taylor-Quinney coefficient of pure aluminium is calculated by the measured results and heat conduction equation. Finally, the distribution of temperature is obtained.

\section{Experimental method}

\subsection{An instrumented Taylor impact test}

The specimen used here is made of pure aluminium A1070. A cylinder with $40 \mathrm{~mm}$ in length and $8 \mathrm{~mm}$ in diameter is manufactured from a received bar. All the specimen is annealed in the atmosphere at $623 \mathrm{~K}$ for $1 \mathrm{~h}$.

Fig. 1 shows a photo of the apparatus based on the instrumented Taylor impact test [1]. The specimen is shot from the launcher shown in bottom left side. A sabot provides a guidance for keeping the straight path of the specimen. The impact velocity is measured by the fiber sensor (KEYENCE FU-77V) fixed at the muzzle of the launcher. In the upper right side of this figure, a Hopkinson pressure bar made of tungsten carbide with $400 \mathrm{~mm}$ in length

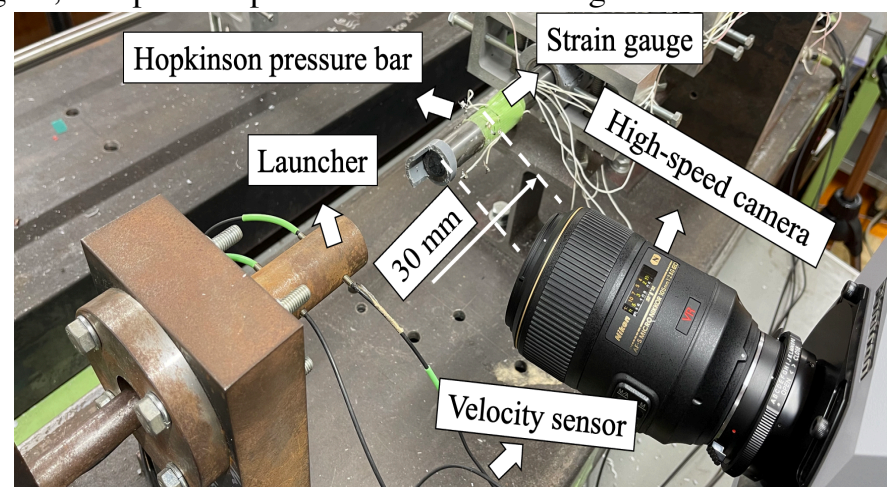

Fig. 1. A photo of the apparatus based on an instrumented Taylor impact test. 
and $20 \mathrm{~mm}$ in diameter is employed to measure the impact force. The elastic deformation of Hopkinson pressure bar is detected by two strain gauges (KYOWA KFG-1-350-D16-11) attached on the surface at $30 \mathrm{~mm}$ away from the impact surface. The deformation process of the specimen is recorded by a high-speed camera (PHOTRON FASTCAM SA-Z) with $210000 \mathrm{fps}$ of frame rate shown in bottom right side of Fig. 1. The air pressure is set to be $0.7 \mathrm{MPa}$. As a result, the impact velocity achieves to $110 \mathrm{~m} / \mathrm{s}$.

\subsection{Temperature measurement system}

Fig. 2 shows a photo of the temperature measurement system using five PIR fibers (ART PHOTONICS M10028) with $860 \mu \mathrm{m}$ in core diameter and an infrared detector (HAMAMATSU P3257-10). Additionally, zinc selenide biconvex lens (THORLABS JAPAN LB7436-E3) with $15 \mathrm{~mm}$ in focal length is placed in front of the detector. As shown in the left hand side of this figure, the temperature measurement is carried out at $5 \mathrm{~mm}$ away from the impact surface. One end of the PIR fiber is fixed radially along with the surface of the specimen. The other ends of the five fibers are bundled and fixed at $15 \mathrm{~mm}$ away from the surface of lens shown in right hand side of Fig. 2. The output voltage of infrared detector is amplified by a signal conditioner and recorded by an oscilloscope. Some details are mentioned in Ref. [13].

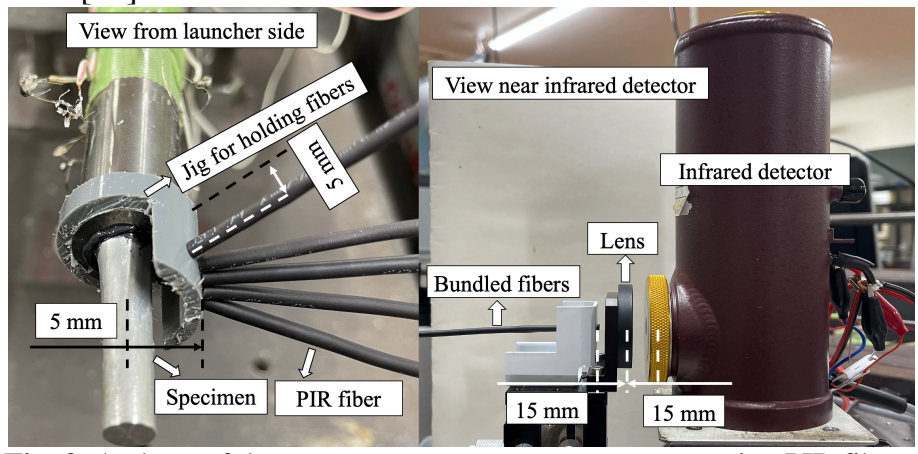

Fig. 2. A photo of the temperature measurement system using PIR fibers.

\section{Calculation of Taylor-Quinney coefficient}

Because of a process under high-speed deformation, the release rate of the generated heat is quite slower than the speed of deformation, the heat conduction equation used here consists the plastic work and thermo-elastic effect. By solving the differential equation of the heat conduction equation, the Taylor-Quinney coefficients $\beta$ can be cauculated as follows.

$$
\beta=\frac{c \rho e^{\gamma \sigma}\left(T-T_{0} e^{-\gamma \sigma}\right)}{\int \sigma e^{\gamma \sigma} d \varepsilon_{p}} \text { and } \gamma=\frac{\alpha_{T}}{c \rho} .
$$

Here, $\sigma$ and $\varepsilon_{p}$ are uniaxial true stress and plastic strain. $\rho, c$ and $\alpha_{T}$ denote the density, the heat capacity and the thermal expansion coefficients with the value of $0.27 \times 10^{4} \mathrm{~kg} / \mathrm{m}^{3}$, $0.913 \times 10^{3} \mathrm{~J} / \mathrm{kg} \cdot \mathrm{K}$ and $24 \times 10^{-6} / \mathrm{K}$, respectively. From Eq. (1), to calculate the $\beta$, the stressstrain curve and the temperature rise at a local point is necessary. From the calculated $\beta$, the distribution of the temperature rise can be obtained by inverse calculation of Eq. (1). Moreover, the calculation method for stress-strain curve is proposed in Ref. [1] 


\section{Experimental results}

Fig. 3 shows the time histories of the impact force and temperature measured by Hopkinson pressure bar and temperature measurement system. In this figure, the blue and orange lines express the impact force and temperature, respectively. The purple and pink dashed lines show the starting time of rising impact force and temperature. Basically, the starting time of temperature will be $4.8 \mu$ s earlier than that of impact force because of the position for attaching the strain gauges considering $6246 \mathrm{~m} / \mathrm{s}$ of the velocity for elastic wave propagation. From this figure, comparing with the starting time of the impact force, $5.8 \mu$ s earlier of the starting time of temperature is observed. Thus, an extremely-high responsiveness for the designed system is confirmed because the starting time for rising the infrared detector signal almost coincides with the prediction. Additionally, the duration for temperature rise is shorter than that for impact force because the deformation process at the measurement position has completed and moved to unloading process.

Fig. 4. shows the stress-strain curve and distribution of temperature rise at $76 \mu$ s of the moment. The blue and red lines show the stress-strain curve and distribution of temperature rise, respectively. After the stress-strain curve at $76 \mu \mathrm{s}$ is obtained by combining the distribution of stress and strain, 1.97 of $\beta$ is calculated by using the stress-strain curve, temperature rise at $76 \mu$ s and Eq. (1). From the calculated $\beta$ and stress-strain curve, the distribution of temperature is calculated as red curve in Fig. 4. From the red curve, it can be observed that the maximum temperature rise is $45 \mathrm{~K}$.

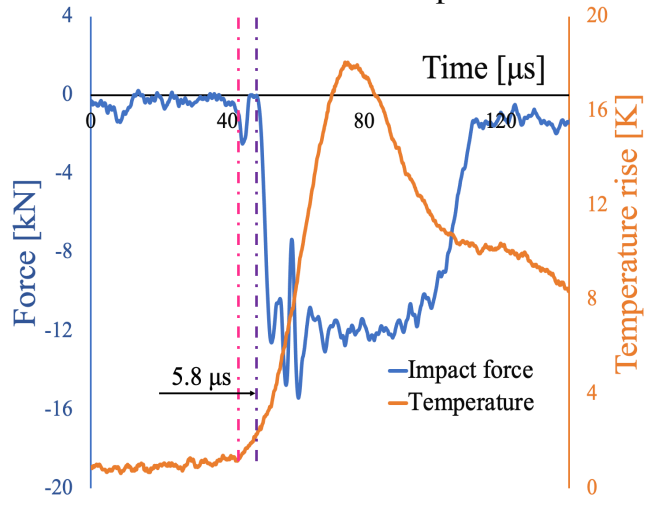

Fig. 3. The time histories of the impact force and temperature rise.

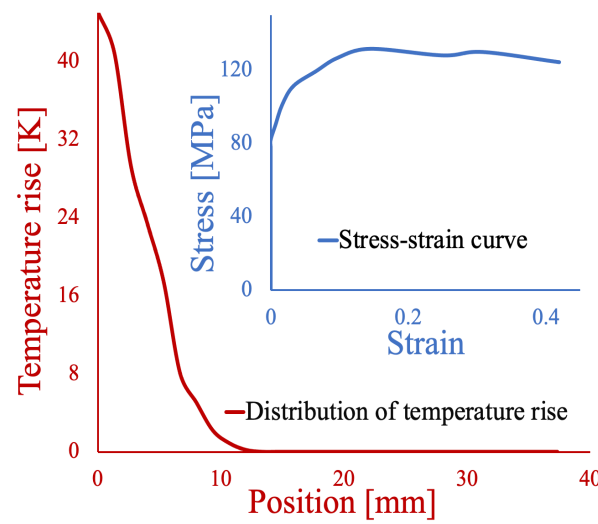

Fig. 4. The stress-strain curve and distribution of temperature rise at $76 \mu$ s of the moment.

\section{Summary}

In this study, an optical measurement system of temperature is established to measure the temperature rise in a local spot-like area on the surface of the specimen during Taylor impact test. Then, the Taylor-Quinney coefficients and distribution of temperature rise is calculated from the heat conduction equation.

\section{Acknowledgements}

We gratefully acknowledge the financial support from JSPS KAKENHI Grant Number JP20H02353 and the Light Metal Educational Foundation, Inc., Japan. Aluminum bars for the specimens are also provided by the Light Metal Educational Foundation, Inc., Japan. 


\section{References}

1. C. Gao and T. Iwamoto, Metals. 8, 642 (2018)

2. J. W. House, B. Aref, J. C. Foster, PP. Gillis, J. Strain. Anal. Eng. Des. 34, 337 (1999)

3. S. L. Lopanikov, B. A. Gama, M. J. Haque, C. Krauthauser, J. W. Gillesspie, M. Guden jr, I. W. Hall, Compos. Struct. 61, 61 (2003)

4. D. Rittel, Exp. Mech. 38, 73 (1998)

5. D. Rittel, Int. J. Solids. Struct. 35, 2959 (1998)

6. B. Cao, T. Iwamoto, Int. J. Impact. Eng. 132, 103284 (2019)

7. H. Kobayashi, F. Okata, K. Ogawa, K. Horikawa, K. Watanabe, J. Soc. Mater. Sci. Jpn. 62, 562 (2013) (In Japanese)

8. G. I. Taylor, H. Quinney, Math. Phys. Eng. Sci. 143, 307 (1934)

9. L. H. Zhang, D. Rittel, S. Osovski, Mater. Sci. Eng. A 729, 94 (2018)

10. D. Rittel, L. H. Zhang, S. Osovski, J. Mech. Phy. Solids. 107, 96 (2017)

11. N. I. Vazquez-Fernandez, G. C. Soares, J. L. Smith, J. D. Seidt, M. Isakov, A. Gilat, V. T. Kuokkala, M. Hokka, J. Dyn. Behav. Mater. 5, 221 (2019)

12. C. Gao, T. Iwamoto, A study on Change in temperature of pure aluminum during impact compressive test by miniature split Hopkinson pressure bar technique, Proceedings of the $10^{\text {th }}$ International symposium on impact engineering ISIE, 2-5 July 2019 Gmunden Austria (2019) 